\title{
Mesocarp and Exocarp of Laffan and Wonderful Pomegran- ate Varieties: By-Products as a Source of Ellagitannins
}

\author{
Mohamad Khatib ${ }^{1}$, Marzia Innocenti ${ }^{1}$, Camilla Giuliani ${ }^{1}$, Amal Al- Tamimi ${ }^{2}$, Annalisa Romani ${ }^{3}$, Nadia \\ Mulinacci $^{1^{*}}$ \\ 'Department of Neuroscience, Psychology, Drug and Child Health, Pharmaceutical and Nutraceutical Section, University of \\ Florence, Via Ugo Schiff 6, Sesto Fiorentino, Florence, Italy \\ ${ }^{2}$ Biology Department of Ecology, Princess Noura University, Al Imam Abdullah Ibn Saud Ibn Abdul Aziz Road, Riyadh, \\ Saudi Arabia \\ ${ }^{3}$ Department of Statistic, Informatics and Applications, “G. Parenti”, Viale Morgagni, 59, Florence Italy
}

*Corresponding author: Nadia Mulinacci, Department of Neuroscience, Psychology, Drug and Child Health, Pharmaceutical and Nutraceutical Section, University of Florence, Via Ugo Schiff 6, Sesto Fiorentino, Florence, Italy,

E-mail:nadia.mulinacci@unifi.it

\begin{abstract}
Pomegranate peel (exocarp and mesocarp), which constitutes about 50\% of the whole fresh fruit, is extensively used in folk medicine but discarded in large quantities as by-product, after the juice production. The objective was to valorize this by-product derived from Laffan and Wonderful varieties, by improving the knowledge on its composition and by applying sustainable extraction processes.

The extraction was performed separately on exocarp and mesocarp, using water at different temperatures, and $70 \%$ ethanol; the ellagitannins were determined by HPLC-DAD and MS- TOF. The polysaccharide fraction was extracted and determined by weight (yields). The profiles of mesocarp and exocarp separately of the two varieties were very similar and characterized by the same ellagitannins (ETs) of pomegranate juice. The highest ETs amount was obtained from mesocarp (140 - 150 $\mathrm{mg}$ /dried matter) via decoction and hydroalcoholic extraction. No ETs degradation was observed during decoction, $\alpha$ and $\beta$ punicalagins were always $59-78 \%$ of the total ETs. Decoction as a green process easy to apply can help to valorize these byproducts as it is able to recover polysaccharides as well as ellagitannins.

A nutritional label was prepared for the whole dry decoction from Wonderful mesocarp.
\end{abstract}

Received Date: April 09, 2017

Accepted Date: June 162017

Published Date: June 222017

Citation: Mulinacci, N., et al. Mesocarp and Exocarp of Laffan and Wonderful Pomegranate Varieties: By-Products as A Source of Ellagitannins. (2017) J Food Nutr Sci 4(2): 1- 7.

DOI: $10.15436 / 2377-0619.17 .1465$

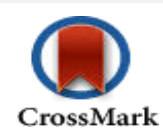

Keywords: Waste; Peel; Punicalagin; Ellagic acid; Decoction; Polysaccharides

\section{Introduction}

Pomegranate (Punica granatum L.) is considered one of the oldest known edible fruits and the Holy Quran, the Bible, the Jewish Torah, and the Babylonian Talmud mention it as a 'Food of Gods' symbolising fertility and prosperity ${ }^{[1]}$. Since antiquity and still today in several countries, different parts of the fruit are used in folk and traditional medicine ${ }^{[1-3]}$. Pomegranate is cultivated around the world in subtropical and tropical regions with different microclimates. Iran currently contributes at least half of the total world production per year (FAOSTAT-FAO, 2010). Other well-known producers are Turkey, Egypt, Spain, Italy and
Syria. Recently, there has been a rise in the global demand for fresh fruit and derived products. The reason for this increased interest in pomegranate is related to the ability of the juice and fruit extracts to promote human health thanks to different mechanisms involving antioxidant, anti-inflammatory, antitumoural and anti-diabetic activities ${ }^{[4]}$. For local populations, not only the juice but also the typical discarded parts such as mesocarp and exocarp, often cited as peel, are used for traditional preparations. In literature it has been reported that pomegranate peel contains Ellagitannins (ETs) and constitutes up to $50 \%$ of the fresh fruit $[2,3,5]$. The peel decoction is used in India, Guatemala, Tunisia, and Syria to treat dysentery, diarrhoea and stomatitis ${ }^{[2]}$. Peel 
extracts, which are popular as antimicrobial mouthwashes ${ }^{[6]}$, also have anthelmintic, antibacterial, antifungal, antimalarial, and antidiarrhoeal properties, as well as respiratory pathologies, haemorrhage, and hypoglycaemic effects ${ }^{[3,7,8]}$.

A recent review shed light on the phytochemical components of peel that have Antihyperglycemic, hepatoprotective, and antihyperlipidemic effects ${ }^{[3,8]}$. Peel extracts have also demonstrated an antimicrobial activity against clinical isolates of pathogenic Staphylococcus aureus and Escherichia coli ${ }^{[9]}$.

According to several authors, $\alpha$ and $\beta$ punicalagins are the main ellagitannins of pomegranate peel, with lesser amounts of ellagic acid and its glycosides ${ }^{[10-13]}$. Nevertheless, the different parts of this fruit have been often incorrectly cited in the scientific works. Indeed, the peel, rightly defined with other synonymous as skin, rind, husk, and hull, is often not differentiated from the mesocarp ${ }^{[10,13,14]}$. A clear example of this mistake is when peel is cited as being $50-65 \%$ of the whole fresh weight of the fruit ${ }^{[2,3]}$. Consequently, little data is available in literature on the separate compositions of pomegranate exocarp and mesocarp. As regards the varieties, although Laffan is widely cultivated in Syria, data on its composition are not available to date. Also called Red Loufani or Lefan, it is known for its sour-sweet flavour, large size, yellow skin, large arils, and very hard seeds ${ }^{[15,16]}$. Laffan is a local strain from Syria, particularly from Idlib (northern Syria), Palestine and Israel, and one of the main varieties cultivated in Hatay, Turkey ${ }^{[17]}$. Conversely, Wonderful is a well-known variety worldwide, grown extensively in California and other Western countries ${ }^{[18]}$. As suggested by some authors $^{[16]}$, Laffan and Wonderful could have a common origin.

The aim of this work was to investigate the distribution of the Ellagitannins (ETs) in the fruit by-products, specifically mesocarp and exocarp of Laffan and Wonderful varieties, by selecting an extraction method for efficiently recovering the ETs and ensuring that was suitable for an easy application on a larger scale. Water was chosen as the elective solvent and compared with an ethanol/water mixture frequently applied to recover different phenols from herbal matrices. A quick HPLC-DAD method was developed to quantify the ETs. The co-presence of polysaccharides was also evaluated in the aqueous extracts and a preliminary nutritional profile determined for decoction from the Wonderful mesocarp.

\section{Materials and Methods}

\section{Samples of pomegranate}

Fresh ripe fruits (close to $10 \mathrm{~kg}$ ) were selected from the Laffan and Wonderful varieties. Laffan cv fruits were harvested from the area of Rif Idlib (Syria) in October 2011; Wonderful fruits were purchased from a local producer, Ortofrutta, Grosseto (Italy) in October 2013. According to the botanical classification, the husk of pomegranate fruit consisting of the exocarp (or rind) and mesocarp (also known as albedo) characterized by a spongy tissue, were collected separately. Exocarp and mesocarp were manually recovered from fresh fruit, washed with water, chopped and freeze-dried. The following abbreviations are used in the text: $\mathrm{M}$ for mesocarp, $\mathrm{E}$ for exocarp, $\mathrm{W}$ for Wonderful and $\mathrm{L}$ for Laffan. The fresh weights of the different parts of the fruit are reported in Table 1 for the two varieties.
Table 1: Weights (\%) of the different parts of fresh fruit; the data are the mean of a triplicate from three fruits.

\begin{tabular}{|l|c|c|c|}
\hline & Seed+Arils & Mesocarp & Exocarp \\
\hline Wonderful & $60.2 \pm 0.1$ & $30.6 \pm 0.44$ & $9.2 \pm 0.34$ \\
\hline Laffan & $55.7 \pm 0.4$ & $36.1 \pm 0.8$ & $8.3 \pm 0.91$ \\
\hline
\end{tabular}

\section{Standard and solvents}

Ellagic acid (purity $\geq 95 \%$ ) and the $\alpha+\beta$ punicalagin mix (purity $\geq 98 \%$ ) were purchased from Sigma Aldrich. All solvents (HPLC grade) and formic acid (ACS reagent) were purchased from Aldrich Company Inc. (Milwaukee, Wisconsin, USA).

\section{Ellagitannin extraction processes}

The dried, finely chopped exocarp and mesocarp were extracted using the same matter/ solvent ratio, $1 \mathrm{~g} / 40 \mathrm{~mL}$. The samples with the prefix Et70 were obtained by adding $70 \%$ ethanol $(\mathrm{v} / \mathrm{v})$ to the dried matter, stirring for $24 \mathrm{~h}$ at room temperature and filtering through what man filter paper to remove the solid residue. The decoction was obtained according to Khatib et al. $(2017)^{[19]}$ by boiling the dried matter in water for $1 \mathrm{~h}$; the supernatant was recovered after cooling and centrifugation (4500 $\mathrm{rpm}, 8 \mathrm{~min}, 4^{\circ} \mathrm{C}$ ), then treated with 2 volumes of ethanol and kept at $0^{\circ} \mathrm{C}$ for $3 \mathrm{hs}$ to induce the polysaccharide precipitation. The solution recovered after centrifugation at $4500 \mathrm{rpm}$ for 12 min was dried to obtain Dec1. The solid residue remaining after filtration was boiled again for $1 \mathrm{~h}$, and treated as described above to obtain Dec2. The precipitated polysaccharides from Dec1 and Dec2 were dried and weighed.

The WEXT sample was derived via water extraction at room temperature and stirring for $24 \mathrm{hrs}$; ethanol was added to the solution recovered, as previously described for decoction, to precipitate the polysaccharides, then the sample was filtered and the solution dried by freeze drying. The dried extracts were re-dissolved in exact volumes of ethanol/water $(7: 3 \mathrm{v} / \mathrm{v})$ and centrifuged at $14000 \mathrm{rpm}$ for $2 \mathrm{~min}$, before the HPLC/DAD analysis.

\section{HPLC-DAD analyses}

The analyses were conducted using an HP 1100L liquid chromatography equipped with a DAD detector (Agilent Technologies, Palo Alto, CA, USA). A $150 \mathrm{~mm} \times 2 \mathrm{~mm}$ i.d., $4 \mu \mathrm{m}$, Fusion RP 80 A, column was used (Phenomenex, USA) with a pre-column of the same phase. The mobile phases were (A) 0.1 $\%$ formic acid/water and (B) $\mathrm{CH} 3 \mathrm{CN}$. The following multi-step linear solvent gradient was used: ${ }^{3}-4 \mathrm{~min} 5-25 \% \mathrm{~B} ; 4-8 \mathrm{~min}$ $25-25 \% \mathrm{~B} ; 8-14 \min 25-35 \% \mathrm{~B} ; 14-16 \min 35-90 \% \mathrm{~B}$ with a final plateau of $2 \mathrm{~min}$ at $5 \% \mathrm{~B}$; total elution time $18 \mathrm{~min}$; flow rate $0.4 \mathrm{~mL} \mathrm{~min}^{-1}$ and oven temperature $26^{\circ} \mathrm{C}$. The UV-Vis spectra ranged from 200 to $500 \mathrm{~nm}$ and the chromatograms were acquired at $240 \mathrm{~nm}, 350 \mathrm{~nm}, 370 \mathrm{~nm}$ and $380 \mathrm{~nm}$. The HPLCDAD and HPLC-MS-TOF experiments were performed on an Agilent 1100 Series HPLC (Agilent Technologies Palo Alto, CA, USA) coupled to an Agilent 6210 TOF mass spectrometer. The accurate mass data of the molecular ions were processed using Data Analysis software (Mass Hunter Work station). Experiments were performed in negative ion mode using a Dual Spray ESI source; the capillary voltage was $4 \mathrm{kV}$. The nebulizer gas was at 20 psi and the drying gas flow and temperature were 6 $\mathrm{L} / \mathrm{min}$ and $350^{\circ} \mathrm{C}$, respectively. The fragment or voltage was set 
at $150 \mathrm{~V}$ to obtain an in-source fragmentation of the ions eluting from the ESI spray. Spectra were recorded at 1.03 spectra/sec and internally calibrated using the reference mass at 922.009798 $\mathrm{m} / \mathrm{z}$. Detection was carried out within a mass range of $80-1600$ $\mathrm{m} / \mathrm{z}$. The chromatographic conditions were the same as described previously.

\section{Quantitative evaluation}

The quantitative evaluation of the main Ellagitannins (ETs) was performed using two external standards: the mix of $\alpha+\beta$ punicalagins at $380 \mathrm{~nm}$ and the ellagic acid at $370 \mathrm{~nm}$. A stock solution of $\alpha+\beta$ punicalagins, $1 \mathrm{mg} / \mathrm{mL}$, was prepared in DMSO and then diluted with $\mathrm{H}_{2} \mathrm{O}$ before the HPLC analyses; a solution of ellagic acid, $0.156 \mathrm{mg} / \mathrm{mL}$, was prepared in $\mathrm{MEOH}$. The calibration curve of $\alpha+\beta$ punicalagins was between $0.5 \mu \mathrm{g}$ and $8 \mu \mathrm{g}$ with an $\mathrm{R}^{2}=0.998$; the calibration curve of ellagic acid was from 0.031 to $1.25 \mu \mathrm{g}$ with $\mathrm{R}^{2}=0.999$.

\section{Results and Discussion}

\section{Extraction processes and yields}

The different pomegranate parts were separated to determine the relative weights (Table 1), obtaining $55-60 \%$ for seeds and arils, 30 - $36 \%$ for mesocarp, and less than $10 \%$ for exocarp. Overall, the mesocarp and exocarp were close to $50 \%$ of the whole fresh fruit for both Laffan and Wonderful. The aim of the extractions applied on mesocarp and exocarp (Scheme 1) was to optimize the extractive yields in terms of ETs, mainly punicalagins and ellagic acid (Figure 1) with respect to the dried fruit, and also to determine the ETs content in the final dried extracts. Simple extractive procedures were selected using water as the suitable solvent for a possible future scale-up, as recently proposed for these by-products by other authors ${ }^{[20]}$. The ETs were chosen as target molecules as they are recognized as the main phenols of pomegranate mesocarp and exocarp ${ }^{[10-13,21]}$. The anthocyanins were not considered in this study for two main reasons. Firstly, our scope was to use water and ethanol/ water only, avoiding acidic media that are required to recover these pigments, as highly unstable in diluted non-acidic aqueous solutions (Toldrà, 2013). Secondly, and in line with previous data $^{[11,21]}$, only the exocarp of pomegranate contains very small amounts (close to $0.2-1.1 \mathrm{~g} / \mathrm{kg}$ dried matter) of the anthocyanins characterizing the arils used to prepare pomegranate juice. Furthermore, the mesocarp, the main discarded part, does not contain these pigments and only a negligible recovery of anthocyanins was expected from our by-products, so they were not evaluated.

The Laffan cv was selected as very widespread in Syria and not investigated to date. At the same time, the Wonderful cv, grown extensively in Western countries, was chosen for comparative purposes, taking into account an earlier work ${ }^{[16]}$ that hypothesized a common origin of these two varieties based on their comparable shape, late ripening and rather sour taste. As early as 1874 , Ibn al-Bitar in his book reported the decoction of pomegranate fruit as a domestic method of preparing a remedy used in traditional Syrian medicine for treating chronic diarrhoea.
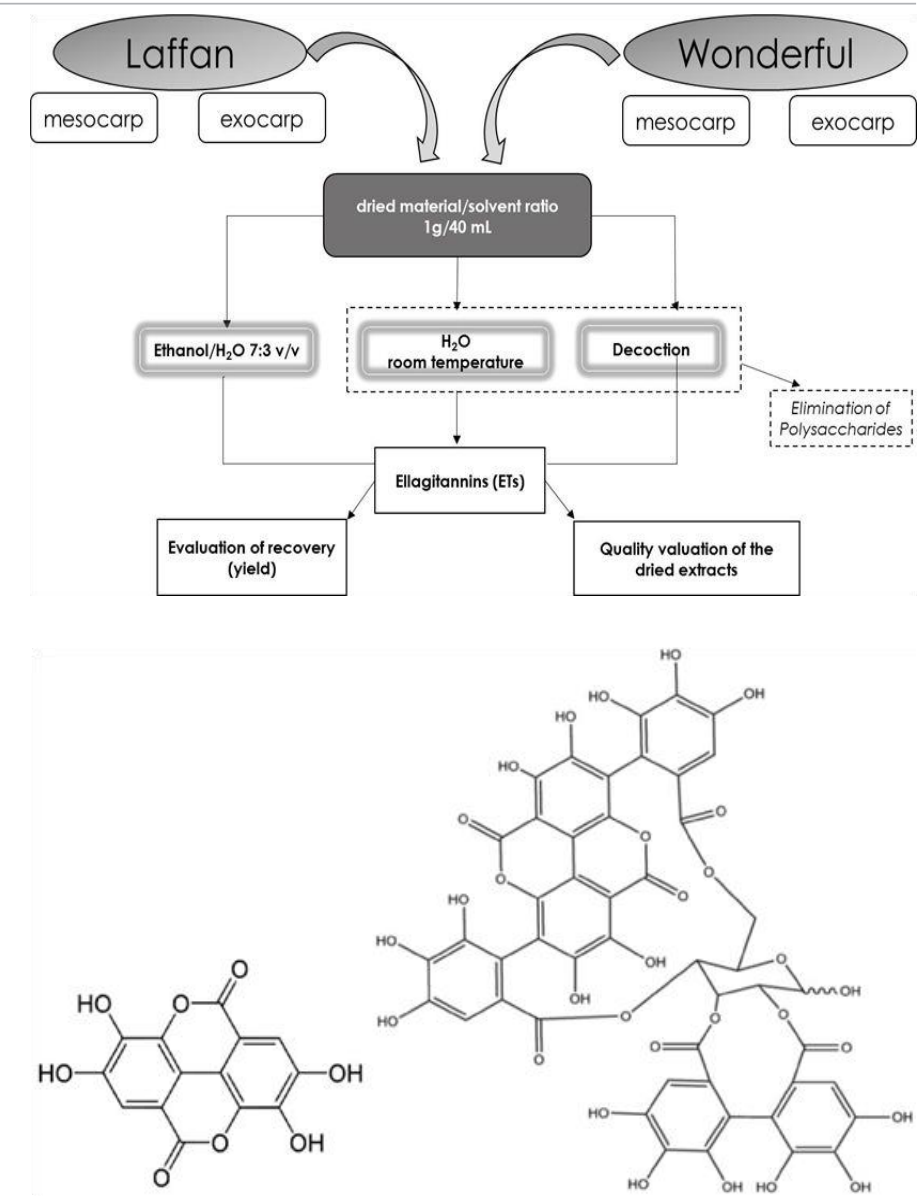

Figure 1: A summary of the extractive methods applied to the fruits of Laffan and Wonderful varieties with the structures of the main pomegranate ETs; ellagic acid (A) and $\alpha+\beta$ punicalagins (B).

In line with this tradition, the decoction was chosen to treat the by-products of the fruit and the results were compared with those from water at room temperature and ethanol/water extractions. The latter mixture with $70 \%$ ethanol is frequently used for the extraction of hydrolysable tannins and other phenols from different herbal products. In the light of our previous results, the water extracts of mesocarp and exocarp were cooled and treated with ethanol to precipitate the polysaccharides $^{[19]}$ before the chromatographic analysis by HPLC.

The use of pure standards $(\alpha+\beta$ punicalagins, ellagic acid), retention times in agreement with previous works ${ }^{[10,11,15]}$, and the mass spectra, made it possible to recognize the ETs as shown in Figure 2. Overall, the chromatographic profiles derived from the different extractions were very similar for the mesocarp and exocarp of the same variety, but also when the two varieties were compared. Our findings showed that pomegranate ETs did not decrease after a long boiling process. The ET content in terms of $\alpha+\beta$ punicalagins, ellagic acid and its glycosides, was expressed on Dried Matter (DM) and summarized in Figure 3. As regards the polysaccharides, few recent papers have reported their presence in pomegranate fruit $^{[22]}$ and our previous findings showed $10 \%$ of polysaccharides in dried Laffan and Wonderful mesocarp, and approximately $5 \%$ in exocarp ${ }^{[19]}$. In the light of these data, the aqueous extracts were treated with ethanol to remove these fractions before the chromatographic analyses. 
This step was not required for the hydro-alcoholic extract owing to the low solubility of the polysaccharides in this medium. Nevertheless, because part of the ETs maybe entrained by the polysaccharides during precipitation, to evaluate this loss the polymers were washed in ethanol/water as previously described (Khatib, 2015), then the solution was recovered, dried and analyzed by HPLC- DAD. During the preparation of Dec1 from mesocarp, the loss of ETs ranged between $11.5 \pm 1.1 \mathrm{mg} / \mathrm{g}$ and $6.47 \pm 0.8 \mathrm{mg} / \mathrm{g}$ of dried matter for Laffan and Wonderful respectively, and was below $4 \mathrm{mg} / \mathrm{g}$ for exocarp. This information could be useful for correctly evaluating the native ellagitannin content in the raw material, but also for indirectly estimating the purity of the recovered polysaccharide fraction. At the end, because the ET amount obtained from the second decoction (Dec2) highlighted about one magnitude order lower than Dec1, a single extraction step with hot water was considered adequate for recovering almost all the ETs (Table 2). In Figure 3, although decoction showed similar efficiency, reaching $100 \mathrm{mg} / \mathrm{g}$, the maximum ET recovery was obtained by ethanol/water extraction $(120 \mathrm{mg} / \mathrm{g})$. Besides, it must be noted that water at room temperature gave similar findings to those from decoction, confirming that pomegranate ETs are thermally stable even after $1 \mathrm{~h}$ of boiling. As regards the use of water as an extractive medium, the results in literature are conflicting.
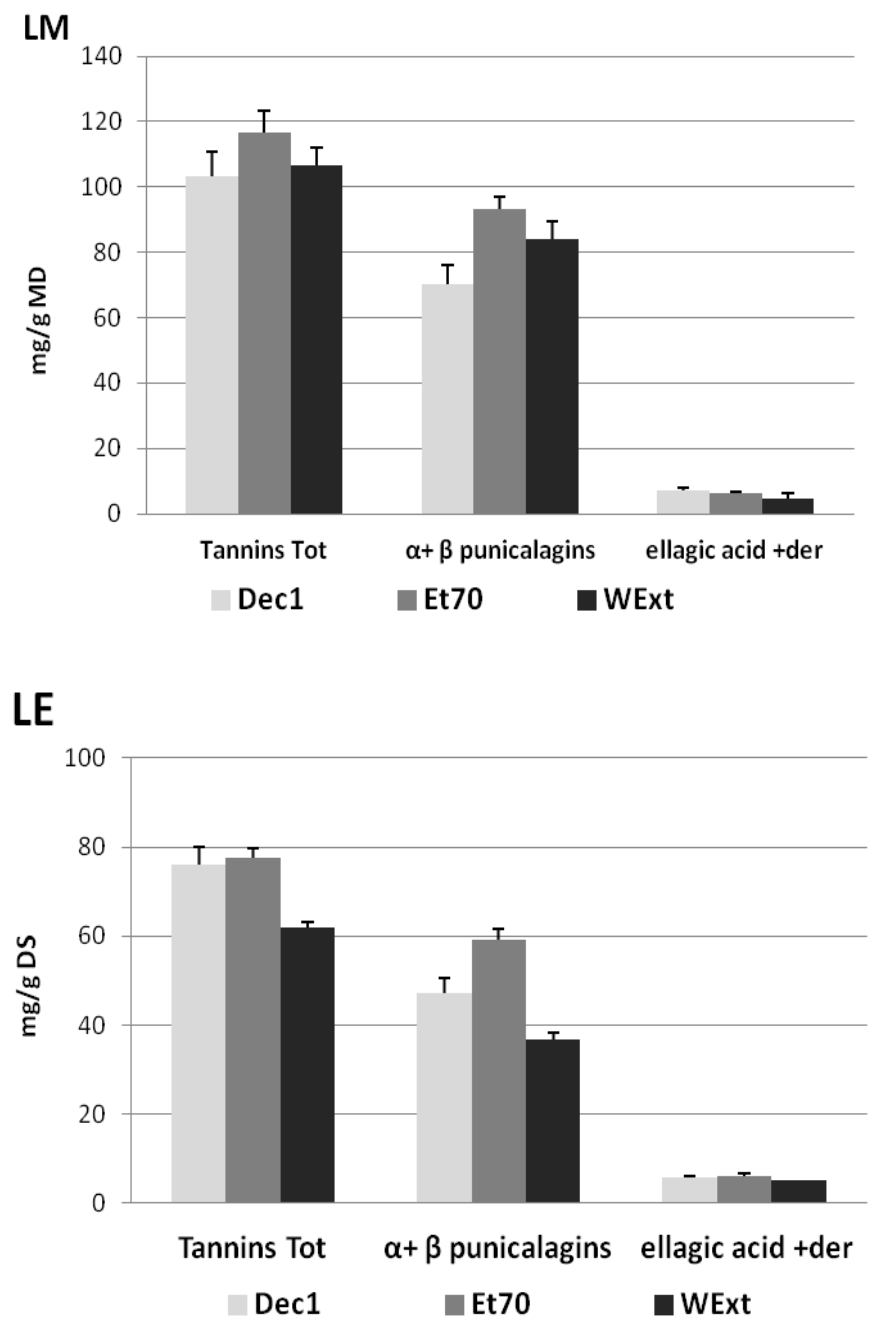

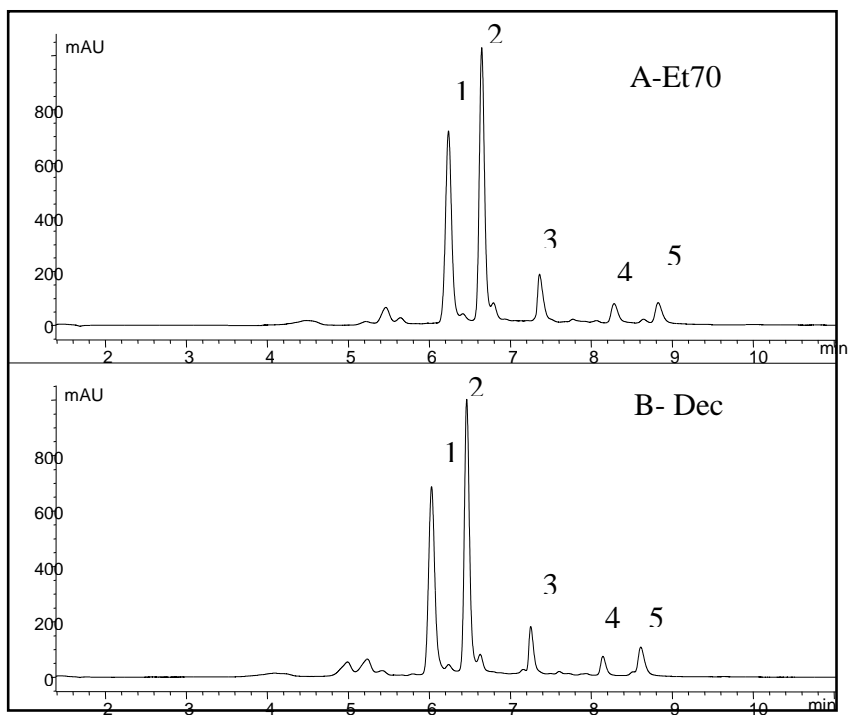

Figure 2: Chromatograms at $370 \mathrm{~nm}$ of the Et70 extracts and Decoction from mesocarp of Wonderful; 1) $\alpha$ - punicalagin, 2) $\beta$ punicalagin, 3) ellagic acid hexoside, 4) ellagic acid pentoside, 5) ellagic acid.
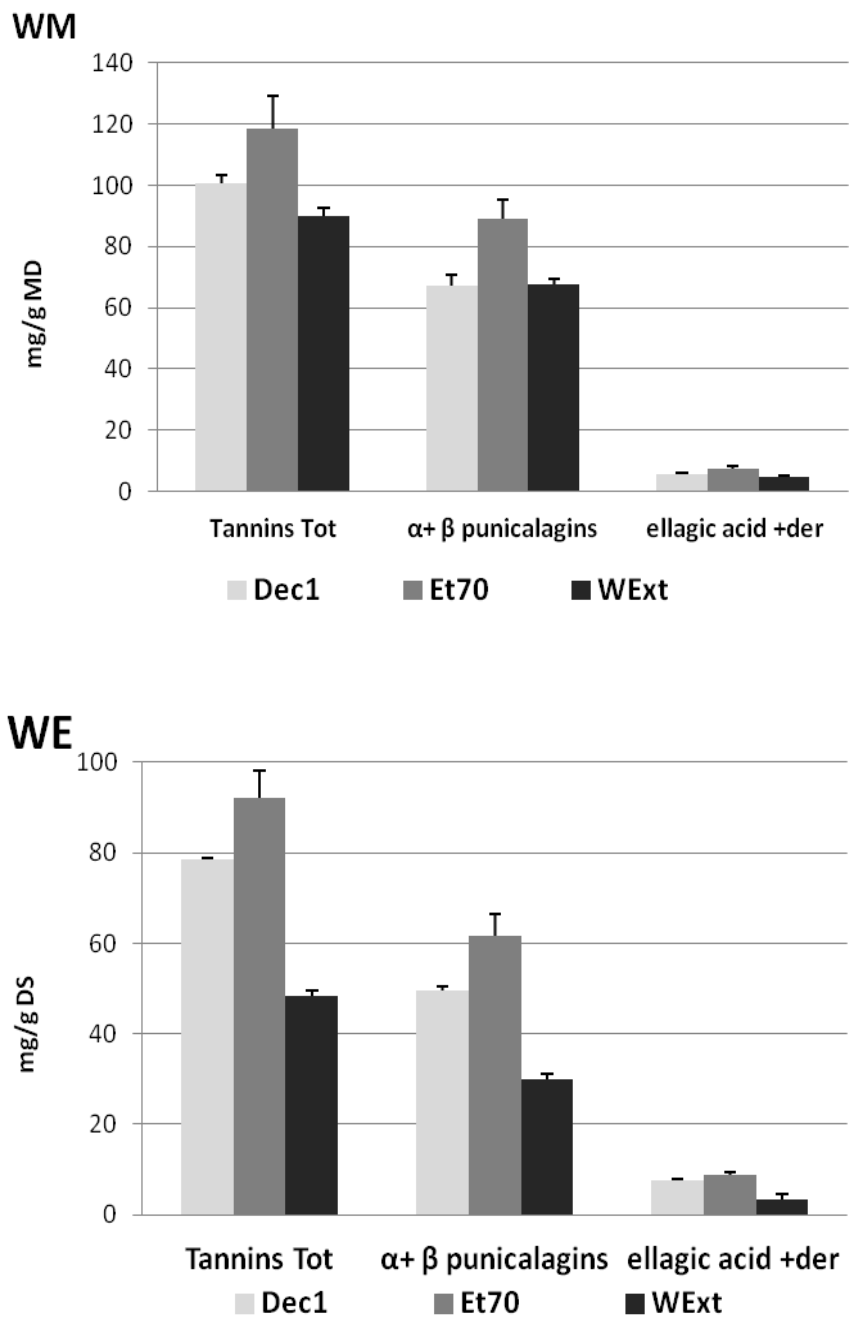

Figure 3: Recovery of the ellagitannins from the different tissues after the selected extractions; data expressed as mg/g of dried tissue as the mean of a triplicate. 
Table 2: Ellagitannins recovered after the second decoction (Dec 2); the data as the mean of a triplicate, are expressed as $\mathrm{mg} / \mathrm{g}$ of dried mesocarp and exocarp.

\begin{tabular}{|c|c|c|c|}
\hline Dec 2 & Compounds & Mesocarp & Exocarp \\
\hline \multirow{4}{*}{ Laffan } & $(\alpha+\beta)$ Punicalagin & $6.25 \pm 1.2$ & $6.33 \pm 0,9$ \\
\cline { 2 - 4 } & $\begin{array}{c}\text { ellagic ac. }+ \text { its } \\
\text { derivatives }\end{array}$ & $1.40 \pm 0.03$ & $1.22 \pm 0.24$ \\
\cline { 2 - 4 } & Total tannins & $10.68 \pm 1,3$ & $11.65 \pm 0.8$ \\
\hline \multirow{3}{*}{ WonderfuL } & \begin{tabular}{c}
$(\alpha+\beta)$ Punicalagin \\
\cline { 2 - 4 }
\end{tabular} & $8.47 \pm 0.1$ & $4.57 \pm 0.01$ \\
\cline { 2 - 4 } & ellagic ac. + its & $1,10 \pm 0.02$ & $1.37 \pm 0.12$ \\
\hline \multirow{2}{*}{ Terivatives } & $13.76 \pm 0.33$ & $8.92 \pm 0.48$ \\
\hline
\end{tabular}

Water at room temperature was less effective than methanol for extracting ETs from pomegranate peel[10,23]. A recent work pointed out how water at room temperature was a less efficient medium in treating pomegranate peel of fruits of different origin when compared to organic solvents like ethanol and ethylacetate ${ }^{[24]}$. Conversely, water was the most effective medium for extracting ETs from dried pomegranate peel, followed by $50 \%$ ethanol $^{[25]}$. As expected from the chromatographic profiles and according to the data in Figure 3, $\alpha$ and $\beta$ punicalagins are the main compounds for all the extracts, ranging between 66 and $78 \%$ of the total ETs. The content of ellagic acid and its derivatives in the mesocarp ranged between 4.5 and $7.3 \%$ of the total ETs, with a higher recovery from hydroalcoholic extraction. A similar trend was observed for the exocarp of both varieties (Table 3). We compared our findings with a study in literature in which an acidified methanol pomegranate extract $(80 \% \mathrm{v} / \mathrm{v})$ from the mesocarp of a Peruvian fruit contained up to $40.6 \mathrm{mg} / \mathrm{g}$ of total ETs and only $0.23 \mathrm{mg} / \mathrm{g}$ of ellagic acid ${ }^{[11]}$. The higher amount obtained in our extracts can be attributed not only to the different fruits, but also to the longer extraction time, more conducive to complete recovery than Fisher's work in which the extraction time was only of $30 \mathrm{~min}$.

Table 3: Content of ellagic acid and its derivatives ( $\mathrm{mg} / \mathrm{g}$ dried matter) in mesocarp and exocarp; the data are the mean of a triplicate of each extraction.

\begin{tabular}{|c|c|c|c|c|c|}
\hline & Extract & cultivar & ellagic ac. hexoside & ellagic ac. pentoside & ellagic ac. \\
\hline \multirow{6}{*}{ mesocarp } & \multirow{2}{*}{ Dec1 } & Laffan & $2.71 \pm 0.22$ & $1.49 \pm 0.07$ & $3.10 \pm 0.33$ \\
\hline & & Wonderful & $2.28 \pm 0.08$ & $1.28 \pm 0.04$ & $2.08 \pm 0.26$ \\
\hline & \multirow{2}{*}{ WExt } & Laffan & $2.17 \pm 0.026$ & $0.89 \pm 0.04$ & $1.70 \pm 0.7$ \\
\hline & & Wonderful & $2.37 \pm 0.21$ & $0.68 \pm 0.02$ & $1.55 \pm 0.21$ \\
\hline & \multirow{2}{*}{ Et70 } & Laffan & $2.76 \pm 0.16$ & $1.34 \pm 0.12$ & $2.10 \pm 0.22$ \\
\hline & & Wonderful & $3.44 \pm 0.07$ & $1.73 \pm 0.27$ & $2.05 \pm 0.02$ \\
\hline \multirow{6}{*}{ exocarp } & \multirow{2}{*}{ Dec1 } & Laffan & $1.64 \pm 0.10$ & $1.54 \pm 0.06$ & $2.73 \pm 0.16$ \\
\hline & & Wonderful & $2.32 \pm 0.03$ & $2.07 \pm 0.03$ & $2.80 \pm 0.07$ \\
\hline & \multirow{2}{*}{ WExt } & Laffan & $1.11 \pm 0.01$ & $1.04 \pm 0.02$ & $2.36 \pm 0.18$ \\
\hline & & Wonderful & $1.10 \pm 0.0$ & $0.72 \pm 0.04$ & $1.61 \pm 0.03$ \\
\hline & \multirow{2}{*}{ Et70 } & Laffan & $2.03 \pm 0.09$ & $1.78 \pm 0.12$ & $1.92 \pm 0.14$ \\
\hline & & Wonderful & $2.86 \pm 0.23$ & $2.89 \pm 0.16$ & $3.14 \pm 0.29$ \\
\hline
\end{tabular}

A study conducted using pressurized hot water to treat pomegranate pee ${ }^{[10]}$ reported an ellagic acid amount of about $1.2 \mathrm{mg} / \mathrm{g}$ dried matter, and similar values for its derivatives corresponding to $2.67-3.5 \%$ of the total ETs. The maximum concentration of ellagic acid $(10.8 \mathrm{mg} / \mathrm{g}$ dried matter) was obtained from the rind of Punica granatum from the Gansu Province in China, refluxed with $50 \%$ methanol $^{[14]}$. It is evident that the lower solubility of ellagic acid in water compared to alcoholic or hydroalcoholic mixtures may negatively affect the recovery of this molecule.

It has been reported that $\alpha$ and $\beta$-punicalagins from Terminalia oblongata when in methanol solvent, interconvert each other with a fixed equilibrium constant $[\beta] /[\alpha]=1$, while this ratio increases up to 4 in a water solution ${ }^{[26]}$. On the contrary, other authors report a fixed ratio of 1.6 in $40 \%$ ethanol $^{[12]}$ and under- score how the ratio does not change depending on the solvent. From our data (Table 4), very similar ratios were observed for the exocarp and mesocarp of the two varieties, with $[\beta] /[\alpha]$ values between $1.2-1.4$ for both, and unmodified ratios in water and $70 \%$ ethanol, in agreement with Lu et al. $(2008)^{[12]}$.
However, it is difficult to compare the data in literature because the composition of the different parts of pomegranate fruit differs greatly depending on variety, growing region, climate, and ripening grade ${ }^{[21]}$, and also because the variety or cultivar are often not reported.

As regards Laffan and Wonderful, our results helped highlight how cheap by-products from pomegranate fruit can be interesting sources not only because they contain the same ETs as the juice, but also because a polysaccharide fraction can be effectively co-extracted simply by using hot water.

\section{Evaluation of the quality of pomegranate extracts}

The quality of the dried pomegranate extracts was evaluated by selecting ETs as the main phytochemical markers, and for the whole decoction (also containing the polysaccharides) was also evaluated its nutritional profile. As can be observed in Figure 4, the different dried extracts from mesocarp had a very similar ET content of around $150 \mathrm{mg} / \mathrm{g}$ of dried extract, while higher amounts, close to $193 \mathrm{mg} / \mathrm{g}$, were from exocarp. As already mentioned, the extracts from the latter showed a higher variability, mainly attributable to the 
inhomogeneous thickness of exocarp containing a residual part of mesocarp. Hypothesizing a larger scale treatment of these byproducts, water is certainly preferable to ethanol mixtures as an extractive medium due to being more economical and easier to handle than other solvents. As previously demonstrated by our group for these varieties[19], hot extraction is also able to guarantee the co-extraction of the polysaccharide fraction. The drying of the whole decoction was easily obtained without adding excipients (e.g. maltodextrins) to reduce the final hygroscopicity, which can be ascribed to the co-presence of the native polysaccharides acting as a natural excipients. Furthermore, the whole polysaccharide fraction of both these varieties showed a prebiotic activity on Bifidobacterium breve B632 and Lactobacillus plantarum L12 strains ${ }^{[19]}$ Table 5 contains a sort of nutritional label plus the ET content for the Wonderful mesocarp decoction. The same evaluation was not possible for the corresponding Laffan extract due to the difficulty in recovering enough fresh fruit for all the analytical determinations during the civil war in Syria. Table 5 shows a fiber content close to $10 \%$ with the soluble fiber as the predominant fraction, a good mineral content indicated by the ashes (5.6\%), and almost $13 \%$ of total ETs.
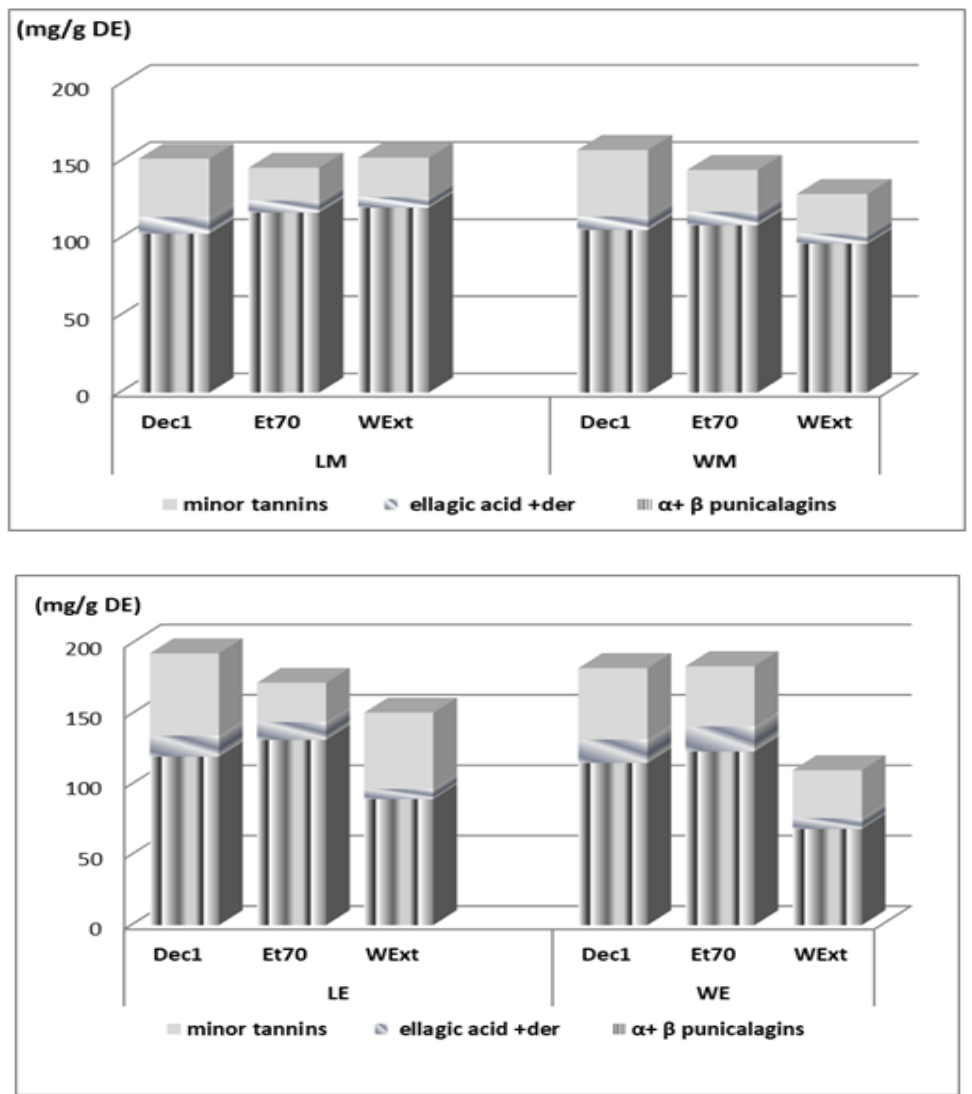

Figure 4: Ellagitannin content (mg/g dried extracts) in mesocarp and exocarp extracts; mean data from a triplicate.

Table 4: Ratios $[\beta] /[\alpha]$ punicalagin for exocarp and mesocarp of the two varieties in the different extracts.

\begin{tabular}{|l|c|c|c|c|}
\hline \multicolumn{5}{|c|}{ mg/g dried matter } \\
\hline Extracts & $\begin{array}{c}\text { Part of the } \\
\text { fruit }\end{array}$ & $\boldsymbol{\alpha}$-punicalagin & $\boldsymbol{\beta}$-punicalagin & $\begin{array}{c}\text { Ratio } \\
{[\boldsymbol{\beta}] /[\boldsymbol{\alpha}]}\end{array}$ \\
\hline \multirow{4}{*}{ Dec1 } & LM & 28.5 & 36.1 & 1.27 \\
\cline { 2 - 5 } & WM & 28.1 & 39.3 & 1.40 \\
\cline { 2 - 5 } & LE & 19.7 & 27.4 & 1.39 \\
\cline { 2 - 5 } & WE & 21.5 & 28.0 & 1.30 \\
\hline \multirow{4}{*}{ WExt } & LM & 36.1 & 47.8 & 1.33 \\
\cline { 2 - 5 } & WM & 27.9 & 39.6 & 1.42 \\
\cline { 2 - 5 } & LE & 15.0 & 18.7 & 1.24 \\
\cline { 2 - 5 } & WE & 13.6 & 16.5 & 1.21 \\
\hline \multirow{4}{*}{ Et70 } & LM & 40.2 & 53.2 & 1.32 \\
\cline { 2 - 5 } & WM & 37.9 & 51.1 & 1.35 \\
\cline { 2 - 5 } & LE & 23.6 & 31.5 & 1.34 \\
\cline { 2 - 5 } & WE & 26.3 & 35.4 & 1.35 \\
\hline
\end{tabular}

Table 5: Nutritional label and ETs content of the whole dried decoction from Wonderful mesocarp.

\begin{tabular}{|l|c|c|}
\hline Determination & $\mathrm{g} / 100 \mathrm{~g}$ & Method \\
\hline Insoluble fiber & 2.99 & AOAC 991.43 \\
\hline Soluble fiber & 6.67 & AOAC 991.43 \\
\hline Proteins & 2.3 & Rapp $\begin{array}{c}\text { Istisan 1996/34 } \\
\text { (Nx6,25) }\end{array}$ \\
\hline Total Reducing Sugars & 43.4 & enzymatic determination \\
\hline Fats & 0.2 & Rapp Istisan 1996/34 \\
\hline ash & 5.6 & $\begin{array}{c}\text { Rapp Istisan 1996/34- meth- } \\
\text { od B }\end{array}$ \\
\hline TotalEllagitannins (ETs) & 12.8 & HPLC-DAD \\
\hline
\end{tabular}

\section{Conclusions}

To evaluate the quality of the dry pomegranate extracts we propose the ellagitannins as main phytochemical markers. The total ellagitannins were comparable in the hydroalcoholic 
and the hot water extracts with the values ranging between 1.5 to $1.9 \%$ dry weights. Even though hydroalcoholic extraction is more effective in recovering the ETs, similar results were also obtained using water as extractive solvent. Water by means of decoction is suggested as a user-friendly and effective method for recovering not only of ETs from pomegranate peel, exocarp and mesocarp, but also the polysaccharide fraction. This fraction was shown to have prebiotic properties and it is fundamental for obtaining a more homogeneous dried extract without the need to add excipients. The compositional profile of the decoction is appropriate for suggesting its use in new food formulations.

Acknowledgments: The research was partially funded by the ECR of Florence within the project, Valorisation of by-products from olive oil production and pomegranate fruit processing (code ECRF15) and by Agenzia Spaziale Italiana (ASI) PAPARD project contract number 2014-34-RO. We thank Dr. Margherita Campo for her contribution within the activities of the EU project LIFE13 ENV IT 000461 / EVERGREEN.

\section{Reference}

1. Khan, G.J., Jamshaid, M., Sajid, M.I., et al. The pharmacological, physiological and toxicological effects of pomegranate fruit extract and its constituents. (2014) Can J Appl Sci 4: 66-80.

Pubmed | Crossref | Others

2. Lansky, E.P., Newman, R.A. Punica granatum (pomegranate) and its potential for prevention and treatment of inflammation and cancer. (2007) J Ethnopharmacol 109(2): 177-206.

Pubmed | Crossref | Others

3. Viuda-Martos, M., Fernández-Lóaez, J., Pérez-álvarez, J.A. Pomegranate and its Many Functional Components as Related to Human Health: A Review. (2010) Compr Rev Food Sci Food Safety 9(6): 635-654.

Pubmed | Crossref | Others

4. Medjakovic, S., Jungbauer, A. Pomegranate: a fruit that ameliorates metabolic syndrome. (2013) Food Funct 4(1): 19-39.

Pubmed | Crossref | Others

5. Akhtar, S., Ismail, T., Fraternale, D., et al. Pomegranate peel and peel extracts: chemistry and food features. (2015) Food Chem 174: 417-425. Pubmed | Crossref | Others

6. Lansky, E., Shubert, S., Neeman, I. Pharmacological and therapeutic properties of pomegranate. (2000) Adv Res Technol 42: 231-235.

Pubmed $\mid$ Crossref $\mid$ Others

7. Swarnakar, Y., Shroff, M., Jhaa, K., et al. Evaluation of Anthelmintic Potential in Fruit Peel of Punica granatum Linn. (Pomegranate). (2013) IJPCS 2: 461-464.

Pubmed | Crossref | Others

8. Middha, S.K., Usha, T., Pande, V. A Review on anti hyperglycemic and Anti hepatoprotective activity of eco-friendly Punica granatum peel waste. (2013) J Evid Based Complement Alternat Med 656172.

Pubmed | Crossref | Others

9. Pagliarulo, C., De, Vito, V., Picariello, G., et al. Inhibitory effect of pomegranate (Punica granatum L.) polyphenol extracts on the bacterial growth and survival of clinical isolates of pathogenic Staphylococcus aureus and Escherichia coli. Food Chem 2016; 190: 824-831.

Pubmed | Crossref | Others

10. Çam, M., Hiş11, Y. Pressurised water extraction of polyphenols from pomegranate peels. (2010) Food Chem 123: 878-885.

Pubmed | Crossref | Others

11. Fischer, U.A., Carle, R., Kammerer, D.R. Identification and quantification of phenolic compounds from pomegranate (Punica granatum L.) peel, mesocarp, aril and differently produced juices by HPLC-DADESI/MSn. (2011) Food Chem 127(2): 807-821.

Pubmed | Crossref | Others
12. Lu, J., Ding, K., Yuan, Q. Determination of Punicalagin Isomers in Pomegranate Husk. (2008) Chromatograph 68(3): 303-306.

Pubmed |Crossref $\mid$ Others

13. Romani, A., Campo, M., Pinelli, P. HPLC/DAD/ESI-MS analyses and anti-radical activity of hydrolyzable tannins from different vegetal species. (2012) Food Chem 130(1): 214-221.

Pubmed | Crossref | Others

14. Zhou, B., Wu, Z., Li, X., et al. Analysis of ellagic acid in pomegranate rinds by capillary electrophoresis and high-performance liquid chromatography. (2008) Phytochem Anal 19(1): 86-89.

Pubmed | Crossref | Others

15. Ozgüven, A.I., Yilmaz, C. Pomegranate growing in Turkey. In : Melgarejo P. (ed.), Martínez-Nicolás J.J. (ed.), Martínez-Tomé J. (ed.). Production, processing and marketing of pomegranate in the Mediterranean region: Advances in research and technology. Zaragoza: CIHEAM, (2000) 41-48 (Options Méditerranéennes : Série A. Séminaires Méditerranéens; n. 42).

Pubmed | Crossref $\mid$ Others

16. Goor, A. The history of the pomegranate in the holy land. (1967) Econ Bot 21(3): 215-230.

Pubmed | Crossref | Others

17. Morton, J. Pomegranate In: Fruits of warm climates. (1987) Morton Collectanea editor, University of Miami, Coral Gables 446-483.

Pubmed $\mid$ Crossref $\mid$ Others

18. Mena, P., Calani, L., Dall'Asta, C., et al. Rapid and comprehensive evaluation of (Poly) phenolic compounds in pomegranate (Punica granatum L.) Juice by UHPLC-MSn. (2012) Molecules 17(12): 1482114840.

Pubmed | Crossref | Others

19. Khatib, M., Giuliani, C., Rossi, F., et al. Polysaccharides from By-products of the Wonderful and Laffan Pomegranate Varieties: New Insight into extraction and characterization. (2017) Food Chem 235: 58-66.

Pubmed | Crossref | Others

20. Goula, A.M., Lazarides, H.N. Integrated processes can turn industrial food waste into valuable food by-products and/or ingredients: The cases of olive mill and pomegranate wastes. (2015) J Food Eng 167: 45-50.

Pubmed | Crossref | Others

21. Fischer, U.A., Jaksch, A.V., Carle, R., et al. Influence of origin source, different fruit tissue and juice extraction methods on anthocyanin, phenolic acid, hydrolysable tannin and isolariciresinol contents of pomegranate (Punica granatum L.) fruits and juices. (2013) Eur Food Res Technol 237(2): 209-221.

Pubmed | Crossref | Others

22. Pereira, P.H.F., Oliveira, T.S., Rosa, M.F., et al. Pectin extraction from pomegranate peels with citric acid. (2016) Int J Biol Macromol 88: 373-379.

Pubmed | Crossref | Others

23. Elfalleh, W., Hannachi, H., Tlili, N., et al. Total phenolic contents and antioxidant activities of pomegranate peel, seed, leaf and flower. (2012) Journal of Medicinal Plants Research 6: 4724-4730.

Pubmed $\mid$ Crossref $\mid$ Others

24. Masci, A., Coccia, A., Lendaro, E., et al. Evaluation of different extraction methods from pomegranate whole fruit or peels and the antioxidant and antiproliferative activity of the polyphenolic fraction. (2016) Food Chem 202: 59-69.

Pubmed | Crossref | Others

25. Zam, W., Ghada, B., Wassim, A., et al. Effective Extraction of Polyphenols and Proanthocyanidins from Pomegranate S Peel. (2012) Int J Pharm Pharm Sci 4: 675-682.

Pubmed | Crossref $\mid$ Others

26. Doig, A.J., Williams, D.H., Oelrichs, P.B., et al. Isolation and Structure Elucidation of Punicalagin, a Toxic Hydrolysable Tannin, from Terminalia oblongata. (1989) J Chem Soc Perkin trans 1: 357-361.

Pubmed | Crossref | Others 